\title{
How Does a Microfluidic Platform Tune the Morphological Properties of Polybenzimidazole Nanoparticles?
}

\author{
Yasin Mehdizadeh Chellehbari ${ }^{1}$, Javad Sayyad Amin ${ }^{2 *}$, Sohrab Zendehboudi ${ }^{3 *}$ \\ ${ }^{1}$ Department of Chemical and Petroleum engineering, Sharif University of Technology, Tehran, IR 11155, Iran \\ ${ }^{2}$ Department of Chemical Engineering, University of Guilan, Rasht, IR 41335, Iran \\ ${ }^{3}$ Department of Process Engineering, Memorial University, St. John's, NL, A1B 3X7, Canada \\ *Email addresses of corresponding authors: sayyadamin@guilan.ac.ir; szendehboudi@mun.ca (+1709-864-2190)
}

\section{S1. METHOD}

For all-atom MD simulations, the bonded and non-bonded parameters of the force field are calculated from the OPLS-AA force field using the polypargen server. OPLS-AA force field is employed to describe the molecular interactions. For MD simulation runs, the total potential energy of simulation cells is defined below:

$E_{\text {tot }}=E_{\text {bond }}+E_{\text {angle }}+E_{\text {dihedral }}+E_{n b}$

where $E_{t o t}, E_{b o n d}, E_{\text {angle }}, E_{\text {dihedral }}$, and $E_{n b}$ stand for the total potential energy and its bond, angle, dihedral, and non-bonded interactions, which are the van der Waals (vdW) and electrostatic forces, respectively. The bonded interactions are designated by their corresponding harmonic forms, and the coulomb potentials and Lennard Jones (LJ) are attributed to the electrostatic and non-bonded vdW interactions. ${ }^{1-4}$

Radius of gyration is an important parameter for NP size analysis. This parameter determines the distance of PBI polymer atoms from their center of mass to the simulation box..$^{5,6}$

Equation (S2) is used to calculate the radius of polymeric cluster in the simulation box. Using Equation (S2), the distance of the atom from the center of mass of the atoms inside the simulation box is calculated. $\mathrm{N}$ refers to number of PBIs in the simulation box.

Radius of Gyration $=\left(\frac{1}{N} \sum\left(d-d_{c m}\right)^{2}\right)^{0.5}$ 
The second measure to evaluate stability in MD simulation is the root mean- square deviation (RMSD), which signifies the deviancy of particle position with respect to the reference location/point at each time. ${ }^{7}$ Equation (S3) is used to determine the RMSD in the simulation box, where $\delta_{\mathrm{i}}$ introduces the space between atom $i$ and either the mean location of the $N$ equivalent atoms or a reference structure. The RMSD results can be confirmed by the gyration radius analysis. ${ }^{8}$ The RMSF parameter introduces a criterion to show the distance between the position of particle $i$ and a reference position, as expressed below: ${ }^{8}$

$$
\begin{aligned}
\operatorname{RMSD} & =\sqrt{\frac{1}{N} \sum_{i=1}^{N} \delta_{i}^{2}} \\
\mathrm{RMSF} & =\sqrt{\frac{1}{T} \sum_{i=1}^{T}\left(r_{i}\left(t_{i}\right)-r_{i}^{r e f}\right)^{2}}
\end{aligned}
$$

in which, $r_{i}^{r e f}$ denotes the reference position of particle $i$ and $T$ represents the time over that one need to average.

The Coulomb energy relations and Lenard-Jones (LJ) energy are used to calculate electrostatic energy and van der Waals (vdW) energy, respectively. ${ }^{1-4,9}$ The determination of electrostatic and vdW energies is based on the average distance between the charges of the atoms and the geometric mean distance of the charges of the atoms from each other, respectively. The LJ energy and Coulomb energy are obtained using Equations (S5) and (S6). The electric charge corresponds to each atom. $K$ is a constant in the Coulomb energy equation. The average total energy at each time is obtained from the simulation using Equation (S7), as given below:

$$
\begin{aligned}
& \text { ENERGY }_{\mathrm{vdW}}=4 \varepsilon\left\{\left(\frac{\sigma}{r}\right)^{12}-\left(\frac{\sigma}{r}\right)^{6}\right\} \\
& \text { ENERGY }_{\text {Electrostatics }}=K^{-1}\left(q_{1} q_{2}\right) / r_{i j}^{2} \\
& \text { ENERGY }_{\text {Total }}=\frac{1}{N} \sum E N E R G Y_{i}
\end{aligned}
$$

Polymer diffusion coefficient can be determined using Equations (S8) and (S9). The coordinate of atoms is shown by " $r$ " and " $t$ " stands for the time. Knowing the magnitude of MSD, the diffusion coefficient can be calculated by using Einstein's equation (e.g., Equation (S9)), as expressed below:

$$
\begin{aligned}
& M S D=(r(t)-r(0))^{2}=\frac{1}{t} \sum_{t=0}^{t}(r(t)-r(0))^{2} \\
& D=\frac{1}{6} \lim _{t \rightarrow \infty} \frac{M S D}{t}
\end{aligned}
$$




\section{REFERENCES}

(1) Jorgensen, W. L.; Tirado-Rives, J. Potential Energy Functions for Atomic-Level Simulations of Water and Organic and Biomolecular Systems. Proc. Natl. Acad. Sci. U. S. A. 2005, 102 (19), 6665-6670. https://doi.org/10.1073/pnas.0408037102.

(2) Siu, S. W. I.; Pluhackova, K.; Böckmann, R. A. Optimization of the OPLS-AA Force Field for Long Hydrocarbons. J. Chem. Theory Comput. 2012, 8 (4), 1459-1470. https://doi.org/10.1021/ct200908r.

(3) Bahlakeh, G.; Nikazar, M.; Hafezi, M. Investigation of the Effects of Methanol Presence on Characteristics of Sulfonated Aromatic Electrolyte Membranes : Molecular Dynamics Simulations. J. Power Sources 2013, 243, 935-945. https://doi.org/10.1016/j.jpowsour.2013.06.066.

(4) Jorgensen, W. L.; Maxwell, D. S.; Tirado-Rives, J. Development and Testing of the OPLS AllAtom Force Field on Conformational Energetics and Properties of Organic Liquids. J. Am. Chem. Soc. 1996, 118 (45), 11225-11236. https://doi.org/10.1021/ja9621760.

(5) Rezaian, M.; Maleki, R.; Dahroud, M. D.; Alamdari, A. PH-Sensitive Co-Adsorption / Release of Doxorubicin and Paclitaxel by Carbon Nanotube, Fullerene, and Graphene Oxide in Combination with N -Isopropylacrylamide: A Molecular Dynamics Study. 1-21. https://doi.org/10.3390/biom8040127.

(6) Bouvier, M.; Liu, F.; Coppalle, A.; Lefevre, G. Impact of Organic Coating on Soot Angular and Spectral Scattering Properties. 2019. https://doi.org/10.1021/acs.est.8b05482.

(7) Sohrabi, S.; Khedri, M.; Maleki, R.; Keshavarz Moraveji, M. Molecular Engineering of the LastGeneration CNTs in Smart Cancer Therapy by Grafting PEG-PLGA-Riboflavin. RSC Adv. 2020, 10 (67), 40637-40648. https://doi.org/10.1039/d0ra07500k.

(8) Maleki, R.; Khedri, M.; Malekahmadi, D.; Mohaghegh, S.; Jahromi, A. M.; Shahbazi, M. A. Simultaneous Doxorubicin Encapsulation and In-Situ Microfluidic Micellization of Bio-Targeted Polymeric Nanohybrids Using Dichalcogenide Monolayers: A Molecular in-Silico Study; Elsevier Ltd., 2021; Vol. 26. https://doi.org/10.1016/j.mtcomm.2020.101948.

(9) Liu, J.; Kong, X.; Jiang, J. Solvent Nano Fi Ltration through Polybenzimidazole Membranes : Unravelling the Role of Pore Size from Molecular Simulations. J. Memb. Sci. 2018, 564 (July), 782-787. https://doi.org/10.1016/j.memsci.2018.07.086. 